REGARDS

SUR L'ECONOMIE ALLEMANDE

BULLETIN ECONOMIQUE DU CRAC

\section{Regards sur l'économie allemande}

Bulletin économique du CIRAC

$70 \mid 2005$

Varia

\title{
Economie informelle : recul du travail au noir
}

Isabelle Bourgeois

\section{OpenEdition}

Journals

Édition électronique

URL : http://journals.openedition.org/rea/2163

DOI : 10.4000/rea.2163

ISBN : 978-2-8218-0836-2

ISSN : 1965-0787

Éditeur

CIRAC

Édition imprimée

Date de publication : 1 mars 2005

ISSN : 1156-8992

\section{Référence électronique}

Isabelle Bourgeois, «Economie informelle : recul du travail au noir », Regards sur l'économie allemande [En ligne], 70 | mars 2005, mis en ligne le 16 décembre 2008, consulté le 15 septembre 2020. URL: http://journals.openedition.org/rea/2163

Ce document a été généré automatiquement le 15 septembre 2020.

(c) CIRAC 


\title{
Economie informelle : recul du travail au noir
}

\author{
Isabelle Bourgeois
}

\section{Le travail dissimulé ne pèsera plus que $15,6 \%$ du PIB en 2005}

1 En 2005, le travail au noir devrait reculer outre-Rhin. Son volume ne représentera plus que 346 milliards $€$ (15,6\% du PIB), soit une baisse de $2,8 \%$ par rapport à 2004 ou de $6,4 \%$ par rapport à 2003. L'économie informelle allemande restera néanmoins dans la moyenne des pays de l'OCDE (15,6\% du PIB également). C'est nettement plus que les 'meilleurs élèves' que sont les Etats-Unis ( $8,2 \%)$, la Suisse (9\%) et l'Autriche comme le Japon $(10,3 \%$, ex aequo). La France $(13,8 \%)$ se montre un peu plus vertueuse que l'Allemagne, et beaucoup plus que les pays sud-européens où l'économie informelle varie entre $21,2 \%$ du PIB (Portugal) et même $27,6 \%$ pour la Grèce, 'championne' en titre.

2 Structurellement, ce sont les secteurs intensifs en main d'œuvre et permettant le paiement en espèces qui apportent la plus forte contribution outre-Rhin (comme ailleurs, au demeurant) : en tête, le BTP et l'artisanat, avec 131,6 milliards $€$, soit $38 \%$ $\mathrm{du}$ total de l'économie informelle. Viennent ensuite, avec 58,9 milliards $€$ (17\%) chacun, l'industrie et le commerce, ainsi que l'hôtellerie et la gastronomie. Le reste se partage entre les 'petits services' comme le baby-sitting ou les leçons particulières (51,8 milliards $€, 15 \%)$ et le secteur du divertissement et des loisirs (45 milliards $€$, $13 \%)$.

\section{L'effet salutaire des mini-jobs...}

3 Les auteurs de ces prévisions, l'économiste Friedrich Schneider (Université de Linz, Autriche) et l'Institut für Angewandte Wirtschaftsforschung (www.iaw.edu) de l'Université de Tübingen, voient dans le recul du travail dissimulé les effets de diverses 
réformes engagées par le gouvernement fédéral. La principale étant la création des mini-jobs (voir REA 67/04), qui aura en quelque sorte 'légalisé' les petits boulots en les exonérant de charges sociales, et se solderait par un recul en volume de 20 milliards $€$ cette année. Viennent ensuite l'assouplissement de la réglementation du travail, comme la hausse à 20 salariés du seuil d'application de la loi de protection contre le licenciement (voir REA 62/03) ou la déréglementation de l'accès à divers métiers dans l'artisanat. Enfin, la réforme de l'impôt sur le revenu pourrait se révéler elle aussi favorable, en rendant du pouvoir d'achat aux ménages, mais aussi par ses effets psychologiques.

\section{... et d'un policy-mix indirect}

4 En revanche, la Loi de Lutte contre l'économie de l'ombre (Gesetz zur Bekämpfung der Schattenwirtschaft), entrée en vigueur en août 2004, n'aurait joué qu'un rôle infime dans la réduction du travail dissimulé (au maximum une baisse de 1,5 milliard $€$ ); tout au plus lui prêtent-ils des effets préventifs. Cette loi avait été adoptée conformément à l'objectif de «réduire la part de l'économie informelle » formulé par la Commission européenne dans ses « lignes directrices » pour une stratégie pour l'emploi adoptées en avril 2003.

5 Le principal obstacle est en effet d'ordre culturel, les Allemands ayant tendance à considérer comme un délit mineur le fait de recourir au travail au noir. Dans ce contexte, le projet de suppression des primes d'accès à la propriété pourrait inciter un certain nombre de ménages à compenser le 'trou' ouvert dans leur budget immobilier en faisant effectuer au noir certains travaux de construction ou d'aménagement. A bien les considérer, ces arguments plaident contre une approche répressive du phénomène, telle que la partagent l'Allemagne et la Suède, et plutôt pour une approche systémique (Espagne ou Italie) tentant d'agir sur l'environnement réglementaire (fiscalité, droit du travail, etc.) à l'origine de l'économie informelle. Or il semblerait que l'Allemagne mêle aujourd'hui ces deux approches, sans qu'il y ait de lien direct entre l'objectif de lutte contre le travail illégal et les réformes adoptées dans le cadre de l'Agenda 2010.

\section{Une réalité qui reste complexe à définir}

Le travail au noir avait connu une forte croissance ces dernières années, outre-Rhin aussi, suscitant une série de débats sur les effets néfastes (distorsion de concurrence, recul des recettes fiscales, déficit des systèmes de protection sociale) ou, à l'inverse, positifs (contribution à la création de richesse), de ces activités non déclarées sur la croissance du PIB 'officiel'. Le pivot de ces débats, déterminant aussi pour la définition d'une politique afférente, est la définition de l'économie informelle.

Dans un ouvrage publié à destination du grand public (Arbeit im Schatten. Wo Deutschlands Wirtschaft wirklich wächst, Gabler, Wiesbaden, 2004), Friedrich Schneider passe en revue les diverses méthodes utilisées pour prendre en compte le phénomène. Il les classe en deux catégories : les méthodes directes, d'un côté, qui se basent sur des sondages d'opinion ou le calcul de la fraude fiscale (niveau microéconomique); de l'autre, les méthodes indirectes (niveau macroéconomique) reposant sur divers indicateurs statistiques (calcul du PIB, du taux d'activité, évolution des transactions 
monétaires ou variations de la consommation d'électricité) et la prise en considération du cadre régissant les activités (degré de réglementation, poids des prélèvements, etc.). Les données comparatives publiées par F. Schneider et l'IAW, et qui alimentent la réflexion de la Commission européenne, mêlent ces deux approches, ce qui explique aussi leurs réserves envers une politique de pure répression, incapable par essence de prendre en compte la complexité des mécanismes générant le travail dissimulé.

\section{0 millions d'emplois en équivalent temps plein}

Selon ces calculs, l'économie informelle n'a cessé de croître outre-Rhin depuis 1975, passant de 5,75\% du PIB 'officiel' à un maximum de 17,4\% en 2003 avant de baisser légèrement depuis. L'unification, avec ses difficultés conjoncturelles, y a certes contribué, mais finalement assez peu en part de PIB : alors qu'en 1990 (ouest de la RFA seulement), l'économie informelle pesait 12,20\%, elle ne représente que $13,90 \%$ en 1995 (est et ouest réunis). La forte diffusion des activités dissimulées outre-Rhin peut se mesurer également en termes d'effectifs occupés: le nombre de travailleurs au noir s'élève à plus de 9 millions en 2004, à quoi il faut ajouter un bon million d'actifs étrangers illégaux. Sachant qu'il s'agit là d'équivalents temps plein, et donc d'une fiction, puisque presque personne ne travaille au noir à temps plein, on mesure l'ampleur de l'économie parallèle, que confirment les vagues successives de sondages présentés dans l'ouvrage. Ainsi, en mai 2003, un quart des Allemands affirmait travailler régulièrement au noir (pour plus de $300 €$ par mois) ; la moitié ( $46 \%)$ avouait recourir régulièrement à des prestations non déclarées.

\section{Une économie dopée par un pouvoir d'achat en recul et les rigidités réglementaires}

Les justifications invoquées en disent long. $90 \%$ des Allemands le font pour dépenser moins, ce qui semble coïncider avec le tassement du pouvoir d'achat (voir REA 67/04) quand on met en regard la troisième raison avancée : "sinon, il faudrait renoncer " (68\%). Contrairement aux apparences, les Allemands ne font donc nullement la 'grève' de la consommation... Les trois quarts $(73 \%)$ font travailler au noir "parce que l'Etat nous prend assez d'argent comme ça ", ce qui confirme le poids des prélèvements obligatoires, mais aussi la faible lisibilité de la fiscalité des ménages, incriminée par le gouvernement fédéral lui-même (voir REA 65/04). La moitié (52\%) des Allemands rappelle "que les entreprises le proposent d'elles-mêmes", ce qui renvoie aux prélèvements, mais aussi à la place éminente du paiement en liquide outre-Rhin. Enfin, $31 \%$ expliquent que ce faisant, "on obtient vite des bons professionnels », ce qui se rapporte à une relative pénurie d'artisans induite par des rigidités corporatistes (voir REA 48/00) ; mais le gouvernement fédéral a commencé à y remédier.

\section{Un réservoir de croissance non négligeable}

Dommageable à la croissance, le travail dissimulé ? Assurément, en termes de manque à gagner pour les budgets publics et le système de protection sociale. Non, si on considère l'étonnante dynamique de l'économie parallèle comme un réservoir potentiel 
de croissance pour le PIB 'officiel'. L'importance de son volume révèle en tout cas que les Allemands mettent à profit le faible nombre d'heures travaillées - pour gagner plus en travaillant plus, mais aussi pour contourner une série de réglementations inhibantes à la fois pour le pouvoir d'achat et l'activité. Reste à savoir comment mobiliser ce potentiel afin qu'il finisse par se traduire dans les statistiques officielles de croissance. (IB)

\section{INDEX}

Mots-clés : économie informelle, emploi, emploi précaire, main d'œuvre, marché du travail, mini-job, travail au noir, travail, travail non déclaré 